\title{
Protocolo de Nagoya: desafíos originados a partir de un texto complejo, ambiguo y controversial
}

\author{
Nagoya Protocol: Challenges Arising \\ from a Complex, Ambiguous and Controversial Text
}

\section{Luciana Carla Silvestri*}

\author{
Sumario: I. Introducción. II. Antecedentes del Protocolo de Nagoya. \\ III. Retos que entraña el Protocolo de Nagoya de cara a su implementación \\ nacional. IV. Conclusiones. V. Bibliografía.
}

* Instituto de Ciencias Humanas, Sociales y Ambientales (INCIHUSA), Mendoza, Argen-
tina. Ha sido investigadora de la Cátedra UNESCO de Territorio y Medio Ambiente de la Uni-
versidad Rey Juan Carlos (Madrid) y es actualmente investigadora en el Instituto de Ciencias
Humanas, Sociales y Ambientales (INCIHUSA-CONICET) (Mendoza, Argentina). Asimismo,
es autora principal-experto para la Evaluación Regional para las Américas de Biodiversidad y
Servicios Ecosistémicos de IPBES (Intergovernmental Platform on Biodiversity and Ecosys-
tem Services), organismo que actúa como brazo científico de Naciones Unidas en temas de
biodiversidad a nivel global. En el pasado (2010-2013) representó a España ante Naciones
Unidas y la Unión Europea en el marco de las negociaciones del Convenio sobre la Diversidad
Biológica y el Protocolo de Nagoya. E-mail:1silvestri@mendoza-conicet.gob.ar.

Artículo recibido el 3 de mayo de 2016 Aprobado para publicación el 22 de septiembre de 2016 
Resumen: El Protocolo de Nagoya sobre acceso a los recursos genéticos y participación justa y equitativa en los beneficios que se deriven de su utilización podría ayudar a lograr varios objetivos, entre ellos: a) conservar la diversidad genética; $b$ ) achicar la brecha tecnológica existente entre el Norte y el Sur y c) compensar a las comunidades indígenas y locales por el uso de su conocimiento tradicional en relación con la utilización de la biodiversidad. Sin embargo, el texto del acuerdo, de carácter complejo, ambiguo y controversial, crea serios desafíos de cara a su implementación. Esta investigación destaca tres: (1) la complejidad, dinamismo y polémica de su ámbito material, (2) las dudas sobre su grado de retroactividad y (3) los problemas que suscita la elección y configuración de las medidas de cumplimiento en relación con su efectividad. Luego de analizar cada reto y aportar algunos elementos aclaratorios sobre ellos, se adelantan posibles soluciones a fin de facilitar la implementación del Protocolo.

Palabras clave: Protocolo de Nagoya, ámbito material, retroactividad, medidas de cumplimiento.

ABSTRACT: The Nagoya Protocol on access to genetic resources and the fair and equitable sharing of benefits arising from their utilization could help conserve genetic diversity, bridge the technological gap between the North and the South, and compensate indigenous and local communities for the use of their traditional knowledge associated to the utilization of biodiversity. However, as the text of the agreement appears complex, ambiguous and controversial, serious national implementation challenges emerge. This research outlines three of them: (1) a complex, dynamic and disputed material scope; (2) doubts on Nagoya's degree of retroactivity and (3) problems in relation to the selection and operationalization of the envisaged compliance measures. After discussing each of the challenges, valuable insights and possible solutions are offered in order to facilitate the national implementation of the Protocol.

Key words: Nagoya Protocol, material scope, retroactivity, compliance measures.

RÉSUMÉ: Le Protocole de Nagoya sur l'accès aux ressources génétiques et le partage juste et équitable des avantages découlant de leur utilisation pourrait contribuer à préserver la diversité génétique, a réduire l' écart technologique entre le Nord et le Sud, et a compenser les communautés autochtones pour l'utilisation de leurs connaissances traditionnelles associées à l'utilisation de la biodiversité. Cependant, le texte de l'accord, de caractère complexe, ambigu et controversé, crée de grands défis en ce qui concerne sa en mise en œuvre nationale. Cette recherche met en évidence trois défis: (1) la complexité, le dynamisme et la controverse qui caractérise son champ d'application matériel; (2) les doutes sur le degré de rétroactivité de ses dispositions et (3) les problèmes qui se déclenchent du choix et de la configuration des mesures d' implementation ayant un impact sur son efficacité. Après avoir analysé chaque défi et de fournir des éléments d'explication à leur sujet, des possibles solutions sont présentées afin de faciliter la mise en œuvre du Protocole.

Mots-clés: Protocole de Nagoya, champ d'application matériel, rétroactivité, mesures de conformité. 
Esta revista forma parte del acervo de la Biblioteca Jurídica Virtual del Instituto de Investigaciones Jurídicas de la UNAM

\section{INTRODUCCIÓN}

El Protocolo de Nagoya ${ }^{1}$ tiene por objetivo la participación justa y equitativa en los beneficios que se deriven de la utilización de los recursos genéticos con el fin de propender a la conservación de la diversidad biológica y la utilización sostenible de sus componentes. ${ }^{2}$ El mecanismo en torno al cual gira, el acceso y distribución de beneficios, más conocido por sus siglas en inglés ABS (access and benefit-sharing), supone grandes dificultades a la hora de su implementación nacional. Algunas de ellas responden al contenido o proceso de desarrollo de regímenes de ABS, como, por ejemplo, la escasa capacidad humana e institucional para diseñarlos y aplicarlos, o la falta de inclusión de las partes interesadas en los procesos de toma de decisión. ${ }^{3}$ Otros en cambio, se originan a partir del texto mismo del Protocolo de Nagoya, el cual resulta por momentos complejo, ambiguo y controversial.

Este artículo identifica y analiza algunos de los retos más destacados de esta última categoría de desafíos mediante el empleo de los métodos analítico y discursivo y un enfoque descriptivo y propositivo. Se enfoca exclusivamente en los desafíos concernientes a la gobernanza de recursos genéticos por haber presentado éstos mayor polémica durante las negociaciones que condujeron a la adopción del Protocolo. ${ }^{4}$ Aporta, asimismo, algunos elementos aclaratorios y adelanta posibles alternativas y soluciones con respecto a cada uno de los retos hallados a fin de facilitar la implementación del Protocolo de Nagoya a nivel nacional.

1 Protocolo de Nagoya sobre acceso a los recursos genéticos y participación justa y equitativa en los beneficios que se deriven de su utilización al Convenio sobre la Diversidad Biológica.

2 Artículo 1o. del Protocolo de Nagoya.

3 Lewis-Lettington, Robert et al., Methodology for Developing Policies and Laws for Access to Genetic Resources and Benefit Sharing, Roma, International Plant Genetic Resources Institute, 2006, p. 2, disponible en: http: / / www.bioversityinternational.org/uploads/tx_news/Methodology_ for_developing_policies_and_laws_for_access_to_genetic_resources_and_benefit_sharing_1150.pdf.

4 El Protocolo de Nagoya también regula el acceso a los conocimientos tradicionales en posesión de comunidades indígenas y/o locales y la participación en los beneficios derivados de la utilización de dichos conocimientos. 
Esta revista forma parte del acervo de la Biblioteca Jurídica Virtual del Instituto de Investigaciones Jurídicas de la UNAM

\section{Antecedentes Del Protocolo De Nagoya}

El Protocolo de Nagoya busca solucionar mediante un mecanismo único, la distribución justa y equitativa de beneficios, tres problemas de naturaleza y origen muy diverso, y de allí en parte, su gran complejidad. Por un lado, pretende palear el declive acentuado y rápido de la biodiversidad, ${ }^{5}$ en este caso genética, y promover su utilización sostenible. ${ }^{6}$ En este contexto, se ha sostenido, no sin discusión, ${ }^{7}$ que la atribución de derechos exclusivos sobre los recursos genéticos ${ }^{8}$ y la consiguiente posibilidad de percibir un "nuevo ingreso económico" a cambio de su acceso, facilitaría la conservación y uso sostenible de los recursos al incrementar su costo de oportunidad. ${ }^{9}$ Por otro lado, el mecanismo de distribución de beneficios ha sido defendido por los países ricos en biodiversidad pero pobres en términos tecnológicos por su virtualidad para achicar la brecha biotecnológica que los separa de los países tecnológicamente más avanzados. Ellos han entendido que era justo beneficiarse de la explotación de sus recursos genéticos tanto como se beneficiaban de la explotación de cualquier otro recurso biológico que se encontraba en su territorio y han revindicado para ese fin, la utilización del mecanismo de acceso y distribución de beneficios. ${ }^{10}$ Por último, el mecanismo de ABS podría ayudar a compensar a las comunidades indígenas y

5 Godt, Christine, “Enforcement of Benefit-Sharing Duties in User Countries”, en Kamau, Evanson C. y Winter, Gerd (eds.), Genetic resources, traditional knowledge \& the law. Solutions for access and benefit sharing, Londres y Sterling (USA), Earthscan, 2009, p. 419.

6 Artículo 1o. del Protocolo de Nagoya.

7 Algunos autores dudan de la eficacia del ABS para lograr la conservación de la biodiversidad, entre ellos: De Jonge, Bram y Louwaars, Niels, "The Diversity of Principles Underlying the Concept of Benefit Sharing”, en Kamau, Evanson C. y Winter, Gerd (eds.), Genetic Resources, Traditional Knowledge \& the Law. Solutions for Access and Benefit Sharing, Londres y Sterling (USA), Earthscan, 2009, p. 51.

8 En este caso se trata de derechos soberanos de las partes sobre sus recursos genéticos.

9 Boisvert, Valerie y Vivien, Franck-Dominique, "The Convention on Biological Diversity: A Conventionalist Approach”, Ecological Economics, vol. 53, núm. 4, 2005, pp. 461-472.

10 De Jonge, Bram y Louwaars, Niels, op. cit., p. 39; Dickson, David y Jayaraman, K. S., “Aid Groups Back Challenge Patent To Neem Patents", Nature, 1995, vol. 377, p. 95; Kloppenburg, Jack Jr., "Biopiracy, Witchery, and the Fables of Ecoliberalism”, Peace Review, 2000, vol. 12, núm. 4, pp. 509-516; Mooney, Pat Roy, "Why I Call it Biopiracy”, en Svarstad, Hanne y Dhillion, Shivcharn (eds.), Responding to Bioprospecting: from Biodiversity in the South to Medicines in the North, Oslo, Spartacus Press, AS, 2000, pp. 37-44. 
Esta revista forma parte del acervo de la Biblioteca Jurídica Virtual del Instituto de Investigaciones Jurídicas de la UNAM

locales que proveen acceso a su conocimiento tradicional asociado a la utilización de la biodiversidad, ya que si bien éste ha sido útil para el progreso de la ciencia y tecnología, nunca ha sido reconocido o retribuido. ${ }^{11}$

El mecanismo de ABS fue por primera vez introducido en el Convenio sobre la Diversidad Biológica (CDB). Allí se reiteró el reconocimiento del principio de soberanía de los Estados sobre los recursos naturales que se encuentran bajo su jurisdicción. ${ }^{12}$ Además se dispuso que las partes tengan la potestad de regular el acceso a sus recursos genéticos, estableciendo que si éstas deciden reglamentarlo, quien desee entonces acceder a ellos deberá contar con el consentimiento fundamentado previo de dicha parte y acordará las condiciones del acceso y uso de recursos genéticos con ella. ${ }^{13}$

Si bien el Convenio adelantó una serie de medidas apropiadas para los Estados proveedores de recursos genéticos, no alumbró nuevas y efectivas medidas de control en las jurisdicciones de las partes donde se utilizaban. Con esto, el problema estaba resuelto a medias ya que no se exigía acreditar en el país del usuario de los recursos genéticos que éstos hubieran sido accedidos legalmente y que se hubieran acordado términos que concretaran la deseada participación en los beneficios. Al mismo tiempo, si bien las Directrices de Bonn ${ }^{14}$ significaron un desarrollo importante para poner efectivamente en marcha las disposiciones sobre ABS previstas en el CDB, sólo constituían una "guía a las partes" y tenían un carácter meramente voluntario, por lo que su efectividad era cuestionada.

De cara a ello, un grupo de países ricos en biodiversidad conformaron el "Grupo de los Países Megadiversos Afines" ${ }^{15}$ y buscaron una nueva instancia

11 Aguilar, Gretel, "Access to genetic resources and protection of traditional knowledge in the territories of indigenous peoples”, Environmental Science \& Policy, vol. 4, núm. 4-5, 2001, pp. 241-256, Schuster, Brian, “A New Integrated Program for Natural Product Development and the Value of an Ethnomedical Approach", The Journal of Alternative and Complementary Medicine, vol. 7, núm. S1, 2001, pp. 61-72.

12 Artículo 3o. del CDB.

13 Artículo 15 del CDB.

14 Directrices de Bonn sobre Acceso a los Recursos Genéticos y Participación Justa y Equitativa en los Beneficios Provenientes de su Utilización. Ellas identifican los pasos en el proceso de acceso y participación en los beneficios, enfatizando la obligación de los usuarios de buscar el consentimiento previo de los proveedores, e individualizan los requisitos principales para negociar términos acordados mutuamente. Si bien las Directrices no son jurídicamente vinculantes, gozan de una autoridad indiscutible al haber sido adoptadas por más de 180 países.

15 Constituido por Brasil, China, Costa Rica, Colombia, Ecuador, India, Indonesia, Kenia, México, Perú, Sudáfrica y Venezuela. 
Esta revista forma parte del acervo de la Biblioteca Jurídica Virtual del Instituto de Investigaciones Jurídicas de la UNAM

negociadora sobre ABS cuyo resultado fuera un régimen que contemplara la certificación de la legal procedencia del material biológico y la existencia del consentimiento fundamentado previo y de los términos mutuamente acordados de transferencia de material genético, como requisitos para la solicitud y el otorgamiento de patentes. ${ }^{16}$ En consideración de lo anterior, el Plan de Implementación de la Cumbre Mundial de Desarrollo Sostenible de 2002 mandó negociar, en el marco del CDB, un régimen internacional para promover y asegurar la distribución justa y equitativa de los beneficios derivados de la utilización de recursos genéticos. ${ }^{17} \mathrm{El}$ proceso de negociación de casi ocho años dio por resultado el Protocolo de Nagoya, adoptado en Nagoya, Japón, el 29 de octubre de 2010 y en vigor desde el 12 de octubre de 2014. ${ }^{18}$

El acuerdo en cuestión dispone que el acceso a recursos genéticos se produzca solamente cuando exista consentimiento fundamentado previo ${ }^{19}$ del país proveedor ${ }^{20} \mathrm{y}$ una distribución justa y equitativa de beneficios entre el solicitante de acceso y el proveedor de los recursos, la cual se concreta mediante condiciones mutuamente acordadas. ${ }^{21}$ Además, obliga a las partes a adoptar diferentes medidas para asegurar que los recursos genéticos utilizados dentro de su jurisdicción hayan sido accedidos de conformidad con el consentimiento fundamentado previo del país proveedor y que se han establecido con él condiciones mutuamente acordadas de acuerdo con su legislación nacional. ${ }^{22}$

16 Documento de la Conferencia de las Partes del Convenio sobre la Diversidad Biológica UNEP/CDB/COP/6/INF/33: Declaración de Cancún de Países Mega-Diversos, disponible en: http://www.cbd.int/doc/meetings/cop/cop-06/information/cop-06-inf-33-es.pdf.

17 Plan de Implementación de la Cumbre Mundial de Desarrollo Sostenible de 2002 de Johannesburgo, Punto 44 (o), disponible en: http: / / www.unctad.org/sp/docs / / aconf1 99d20\&c1_ sp.pdf.

18 El Protocolo de Nagoya contaba con 70 partes el día 8 de abril de 2016, disponible en: https: / / www.cbd.int/abs/nagoya-protocol/signatories/default.shtml.

19 El consentimiento fundamentado previo se refiere al permiso o autorización otorgada luego de haber sido informado de manera completa sobre todos los extremos necesarios para realizar una decisión sopesada. Debe manifestarse por escrito en un documento oficial que identifica el origen in situ de los recursos genéticos y autoriza el acceso a los mismos.

20 Artículo 6.1 del Protocolo de Nagoya.

21 Artículo 5.1 del Protocolo de Nagoya.

22 Artículos 15, 17 y 18 del Protocolo de Nagoya. 
Esta revista forma parte del acervo de la Biblioteca Jurídica Virtual del Instituto de Investigaciones Jurídicas de la UNAM

\section{RETOS QUE ENTRAÑA EL PROTOCOLO DE NAGOYA DE CARA A SU IMPLEMENTACIÓN NACIONAL}

\section{La complejidad, dinamismo y polémica del ámbito material}

El ámbito material del Protocolo, aplicable a recursos genéticos y beneficios que se deriven de la utilización de dichos recursos, ${ }^{23}$ presenta retos para su implementación nacional debido a tres cuestiones. Primero, por su complejidad. Tómese como ejemplo las nociones de "recursos genéticos" o de "derivados". Ellas no sólo son realidades ásperas y lejanas al entendimiento de la persona común, sino también a la comprensión de la mayoría de los funcionarios encargados de la implementación del Protocolo a nivel nacional y de los demás actores involucrados en el ABS. Ellos difícilmente ostentan al mismo tiempo titulaciones en biología, química, biotecnología y derecho, por sólo mencionar algunas de las ramas del saber que guardan relación con la aplicación del Protocolo. Tampoco tienen la posibilidad real de profundizar sobre estos conceptos, sea por escasez de tiempo o por la prioridad que presentan otros asuntos ambientales que gestionan; con lo cual es frecuente toparse con funcionarios bienintencionados pero abrumados ante la tarea de implementar regímenes que regulan elementos complejos y que poco conocen.

En segundo lugar, los elementos que el Protocolo de Nagoya pretende regular son dinámicos, ${ }^{24}$ estando sujetos a continuos cambios que responden al avance de la ciencia y la tecnología y a la viabilidad comercial devenida de ciertos productos y servicios biotecnológicos. Ante tal dinamismo, el material genético que ayer no tenía valor ni real ni potencial, mañana podría tenerlo, y encontrarse por tal motivo, incluido en el ámbito mate-

23 Artículo 3o. del Protocolo de Nagoya. El Protocolo también es aplicable a los conocimientos tradicionales en posesión de las comunidades indígenas y/o locales y a los beneficios que surjan de la utilización de dichos conocimientos, pero estos elementos no son tratados en este artículo.

24 Documento del Grupo de Trabajo Especial de Composición Abierta sobre Acceso y Participación en los Beneficios UNEP/CBD/WG-ABS/9/INF/1: The Concept of "Genetic Resources" in the Convention on Biological Diversity and How it Relates to a Functional International Regime on Access and Benefit-Sharing, disponible en: https: / / www.cbd.int/doc/ meetings/abs/abswg-09/information/abswg-09-inf-01-en.pdf. 
Esta revista forma parte del acervo de la Biblioteca Jurídica Virtual del Instituto de Investigaciones Jurídicas de la UNAM

rial del Protocolo. Supone por lo tanto un gran reto intentar cubrir en los regímenes nacionales realidades en continuo cambio.

En tercer lugar, y relacionado con lo anterior, el ámbito material del Protocolo resulta polémico: la inclusión o exclusión de determinadas situaciones del régimen regulado por el Protocolo de Nagoya responde a largas negociaciones internacionales que ponen de manifiesto la diversidad de intereses que existe entre los países del Norte y del Sur. ${ }^{25}$ Esta diferencia no acaba con un acuerdo sobre el texto del Protocolo, sino que muchas veces continúa y se cuela en las legislaciones nacionales que se adoptan en su cumplimiento. Por ejemplo, si bien existe acuerdo en que los derivados están comprendidos por el régimen internacional, hay desacuerdo sobre si éstos abarcan sólo la estructura genética o también cubren la información genética que pueda ser leída, digitalizada y almacenada en bancos de datos.

Considerando lo anterior, resulta imprescindible aportar algunas aclaraciones sobre el ámbito material del Protocolo. Los recursos genéticos cubiertos por éste son los que se encuentran incluidos en el CDB. ${ }^{26}$ Por lo tanto, queda comprendido todo material de origen vegetal, animal, microbiano o de otro tipo que contenga unidades funcionales de la herencia con valor real o potencial, ${ }^{27}$ quedando incluidas las semillas, el esperma, organismos individuales, células, cromosomas, genes, plásmidos o cualquier parte de éstos. ${ }^{28}$

Ese material para ser considerado "genético" debe contener "unidades funcionales de la herencia” y éste es el quid de la cuestión. Por "unidades de la herencia" se entiende las unidades estructurales moleculares de ácido desoxirribonucleico (ADN) y el ácido ribonucleico (ARN). ${ }^{29}$ Ellas abarcan dos tipos de dimensiones o valores: la estructura genética en sí misma y la información

25 Tsioumani, Elsa, “Access and Benefit Sharing: the Nagoya Protocol”, Environmental Policy and Law, 2010, vol. 40, núm. 6, pp. 288-293.

26 Artículo 3o. del Protocolo de Nagoya.

27 Párrafos 10 y 15 del artículo 2o. del CDB.

28 Queda excluido cualquier tipo de material genético de origen humano. Decisión II/11 punto 2, de la Conferencia de las Partes del Convenio sobre la Diversidad Biológica, disponible en: http://www.cbd.int/decision/cop/default.shtml?id=7084.

29 Documento del Grupo de Trabajo Especial de Composición Abierta sobre Acceso y Participación en los Beneficios UNEP/CBD/WG-ABS/7/2: Report of the Meeting of the Group of Legal and Technical Experts on Concepts, Terms, Working Definitions and Sectoral Approaches, disponible en: https://www.cbd.int/doc/meetings/abs/abswg-07/official/abswg07-02-en.pdf. 
almacenada en la secuencia de nucleótidos que puede ser leída, digitalizada y almacenada. ${ }^{30}$ Ambos valores/funciones quedan comprendidos entonces en el régimen internacional de ABS. Sin embargo, tal opinión no es uniforme y contrariamente se ha sostenido que por "unidades de la herencia" sólo debe entenderse el sustrato de material genético y no la información científica intangible que hace al funcionamiento genético. ${ }^{31}$ Posicionarse sobre la cuestión resulta fundamental porque la información genética de interés podría encontrarse sólo almacenada en una base de datos, y su utilización, dependiendo del punto de vista que se adopte, desencadenaría o no la aplicación del régimen de distribución de beneficios con el país que proveyó el recurso genético de conformidad con el Protocolo.

Por otra parte, las unidades de la herencia deben ser "funcionales"; es decir, deben servir o satisfacer una necesidad. Por tanto, y esto es necesario destacarlo, "funcional," no significa que la unidad pueda reproducirse a sí misma, sino que pueda ser objeto de manipulación genética. ${ }^{32}$ Asimismo, el material genético en cuestión debe tener un "valor real o potencial". Eso sugiere que debe existir una atribución de valor por parte de un sujeto y que este valor depende del estado de la técnica en un momento dado; de modo que los recursos genéticos que hoy no tienen un valor en especial, podrían tenerlo en el futuro gracias al avance de la ciencia.

Por último, es necesario aclarar que, lo que desencadena la aplicación del régimen de ABS previsto en el Protocolo es el uso que se le dará al material. Así, productos como la madera o la lana que contienen $\mathrm{ADN}$ podrían quedar comprendidos en el régimen de ABS si ellos pretenden ser usados como material genético y no como materias primas en procesos industriales más convencionales. De hecho, se estima que en el corazón de la definición de recursos genéticos se hallan dos elementos: el primero se refiere al "código genético" o características genéticas únicas de una especie, y el segundo, al "uso" del código genético o a la información científica referida a esas características. ${ }^{33}$ Por lo tanto, actividades tales como la modificación genética

30 Documento UNEP/CBD/WG-ABS/9/INF/1 ... op. cit.

31 Winter, Gerd, “Towards Common Pools of GRs-Improving the Effectiveness and Justice of ABS”, en Kamau, Evanson C. y Winter, Gerd (eds.), Genetic Resources, Traditional Knowledge \& the Law. Solutions for Access and Benefit Sharing, Londres y Sterling (USA), Earthscan, 2009, p. 22.

32 Idem.

33 Chishakwe, Nyasha y Young, Tomme Rosanne, Access to Genetic Sesources, and Sharing the 
Esta revista forma parte del acervo de la Biblioteca Jurídica Virtual del Instituto de Investigaciones Jurídicas de la UNAM

para el desarrollo de nuevas especies, la conservación de recursos genéticos con fines de conservación o de reintroducción, o la producción de compuestos que ocurren naturalmente en el material genético se consideran incluidas dentro del régimen de ABS. ${ }^{34}$

El Protocolo de Nagoya también comprende dentro de su ámbito material "los beneficios que se deriven de la utilización de los recursos genéticos". ${ }^{35}$ Ni el Protocolo ni el Convenio incluyen una definición de "beneficios"; sin embargo, por ellos se puede entender las ventajas que emanan de la utilización de los resultados de la investigación biotecnológica como, por ejemplo, la información técnica o tecnológica, las ganancias obtenidas, los royalties, etcétera. ${ }^{36}$ La falta de definición es suplida por un listado ejemplificativo de los beneficios monetarios y no monetarios que pueden negociarse en el marco del ABS y que es presentado como anexo al final del Protocolo.

Por el contrario, el Protocolo sí define el concepto de "utilización de recursos genéticos". ${ }^{37}$ De una interpretación integral del texto del Protocolo surge que éste es aplicable a todos los beneficios provenientes de la utilización de los recursos genéticos, surjan éstos de la investigación o desarrollo sobre la composición genética o la composición bioquímica de los mismos, sea cual sea la técnica o tecnología que se use, encontrándose incluida la biotecnología como medio para alcanzar esos beneficios. ${ }^{38}$ La inclusión de la biotecnología como forma o medio de obtener esos beneficios abre la puerta a aquéllos surgidos de la aplicación tecnológica que utiliza sistemas biológicos, organismos vivos y derivados para la creación o modificación de productos o procesos para usos específicos. ${ }^{39}$

Y para que no cupieran dudas, el Protocolo de Nagoya incluyó expresamente a los derivados en su ámbito material. ${ }^{40}$ Ejemplos de éstos son los aromas usados por la industria cosmética y los bioquímicos útiles para el

Benefits of their Use: International and Subregional Issues [Informe inédito], IUCN, 2003. p. 5, disponible en: http://weavingaweb.org/absdocuments/eng_SADC.pdf.

34 Documento UNEP/CBD/WG-ABS/7/2 ...op. cit.

35 Artículo 3o. del Protocolo de Nagoya.

36 Glowka, Lyle et al., A Guide to the Convention on Biological Diversity, Gland y Cambridge, IUCN, 1994, p. 83.

37 Al hacerlo, el artículo 3o. remite al artículo 2o. incisos c, d y e del Protocolo.

38 Artículo 2.c Protocolo Nagoya.

39 Artículo 2.d del Protocolo de Nagoya.

40 Artículo 2.e del Protocolo de Nagoya. 
sector farmacéutico. Al igual que para el caso de los recursos genéticos, es importante aclarar que se estima que los "derivados" en el marco del Protocolo comprenden dos tipos de dimensiones: la estructura genética en sí misma y la información almacenada en la secuencia de nucleótidos que puede ser leída, digitalizada y almacenada, ${ }^{41}$ ya que se piensa que el Protocolo ha querido incluir ambos valores al disponer que "derivado [comprende] un compuesto bioquímico... incluso aunque no contenga unidades funcionales de la herencia", ${ }^{42}$ es decir, aunque no esté contenido en el material genético. ${ }^{43}$ Otros en cambio, entienden que éstos sólo incluyen la primera dimensión, ${ }^{44}$ y que en todos los casos, para que sea aplicable el Protocolo, debe tratarse de un acceso a recursos genéticos que involucre una "materialidad".

\section{Dudas sobre la retroactividad de las normas}

El Protocolo de Nagoya no dispone su grado de retroactividad. Dicho silencio puede convertirse en un gran desafío para la implementación nacional del Protocolo de Nagoya en varias situaciones. Una por ejemplo, se configura con respecto a la posibilidad de acceder al sistema jurisdiccional de la parte donde han sido utilizados los recursos genéticos, alternativa que ahora sí cubre el Protocolo de Nagoya, ${ }^{45}$ en caso de un acceso ilegal a recursos genéticos acaecido con anterioridad al Protocolo.

Durante las negociaciones se propusieron diversos ámbitos temporales, presentándose la cuestión como uno de los temas más espinosos de cara al gran número y riqueza de colecciones ex situ emprendidas con anterioridad

41 Documento UNEP/CBD/WG-ABS/9/INF/1 ... op. cit.

42 Artículo 2.e del Protocolo de Nagoya.

43 Singh Nijar, Gurdial, "The Nagoya Protocol on Access and Benefit Sharing of Genetic Resources: Analysis and Implementation Options for Developing Countries”, South Center Research Papers, 2011, núm. 36, pp. 1-38, disponible en: http://www.southcentre.int/wp-content/ uploads/2013/05/RP36_The-Nogoya-Protocol_EN.pdf.

${ }_{44}$ Buck, Matthias y Hamilton, Clare, "The Nagoya Protocol on Access to Genetic Resources and the Fair and Equitable Sharing of Benefits Arising from their Utilization to the Convention on Biological Diversity", Review of European Community and International Environmental Law, vol. 20, núm. 1, 2011, pp. 47-61.

45 Artículo 18.3.a Protocolo de Nagoya. 
Esta revista forma parte del acervo de la Biblioteca Jurídica Virtual del Instituto de Investigaciones Jurídicas de la UNAM

a la vigencia del Convenio. ${ }^{46}$ En general, los países en vías de desarrollo favorecieron la aplicación de las normas del Protocolo a accesos producidos con anterioridad al mismo y con posterioridad al Convenio; mientras que los países desarrollados preferían que el Protocolo no tuviera ningún grado de retroactividad. ${ }^{47}$ Como se dijo, el Protocolo de Nagoya no se decantó por ninguna opción.

Afortunadamente, la Convención de Viena sobre el Derecho de los Tra$\operatorname{tados}^{48}$ ayuda a aportar luz sobre el tema. Ella establece la irretroactividad de los tratados internacionales entre Estados con respecto a actos o hechos que haya tenido lugar con anterioridad, o que hayan dejado de existir, a la fecha de entrada en vigor del tratado con respecto a la parte en cuestión. ${ }^{49}$ De allí se desprende que en el marco internacional rige el principio de irretroactividad y que por tanto, las disposiciones del Protocolo no son aplicables, por ejemplo, a accesos a recursos genéticos y reparto de los beneficios derivados de su utilización que se hayan producidos con anterioridad a su entrada en vigor.

Ahora bien, una situación confusa ocurre cuando los beneficios de la utilización de los recursos genéticos se producen con posterioridad a la entrada en vigor del Protocolo de Nagoya, pero el acceso a los mismos ha sido anterior a él, aunque posterior al Convenio. ¿Estamos aquí ante un hecho nuevo (obtención de beneficios) en el sentido de la Convención de Viena? ¿Se podría aplicar por tanto retroactivamente el Protocolo de Nagoya y exigir reparto de beneficios en estas situaciones? Algunos creen que sí, ${ }^{50}$ porque los beneficios son actos nuevos o continúan, por lo que no se aplicaría el principio de irretroactividad. Eso significa que para el caso particular de recursos genéticos accedidos con posterioridad al CDB, que hubieran sido obtenidos de países donde sí existían marcos nacionales ${ }^{51}$ y cuyos beneficios (usos continuos y/o nuevos) se producen con posterioridad a la

46 Buck, Matthias y Hamilton, Clare, op. cit., p.50 y ss.

47 Singh Nijar, Gurdial, op. cit., p. 19 y ss.

48 Codifica el derecho internacional consuetudinario de los tratados.

49 Artículo 28 de la Convención de Viena sobre el Derecho de los Tratados.

50 Singh Nijar, Gurdial, op. cit., p. 19 y ss., Greiber, Thomas et al., Guía explicativa del Protocolo de Nagoya sobre Acceso y Participación en los Beneficios, Gland (Suiza), UICN, 2012, p. 79.

51 Los marcos nacionales del país proveedor de recursos genéticos tienen que haber existido al momento del acceso y deben haber exigido la distribución de beneficios, de lo contrario se vulneraría el principio de irretroactividad de la ley y el principio de legalidad. 
entrada en vigor del Protocolo, el mismo sí sería aplicable. Quienes opinan así entienden que el Protocolo es una mera especificación de obligaciones ya existentes en el Convenio, sobre todo de los artículos 15.5 y 15.7, por lo cual, negar la retroactividad del Protocolo para los supuestos de usos nuevos y continuados significaría sanear la ilegalidad de accesos producidos en violación a lo dispuesto por estos artículos, resultando esto inaceptable. Una interpretación en este sentido incentivaría a las partes a ratificar el Protocolo lo más tarde posible para seguir de esa manera accediendo a recursos genéticos sin necesidad de obtener consentimiento fundamentado previo y sin necesidad de repartir beneficios en el interim, ${ }^{52}$ es decir, incumpliendo impunemente la normativa nacional de aquellos países parte del CDB que han sido serios y rigurosos y se han molestado en articular y poner en marcha marcos nacionales de acceso que han favorecido la seguridad jurídica. Otros, en cambio, creen que se trata de una zona de grises de difícil solución y que plantear la retroactividad de la norma internacional puede conllevar a crear mayor inseguridad jurídica. ${ }^{53}$

Se piensa que esta espinosa cuestión debería ser zanjada definitivamente por las sucesivas Decisiones que la Conferencia de las Partes adopte. En cualquier caso, y más allá de ellas, una manera efectiva de solucionar el conflicto en la práctica respecto a las colecciones ex situ ya existentes, es a través de la adopción voluntaria de códigos y políticas de conducta que vayan en consonancia con el espíritu y la letra del CDB y del Protocolo de Nagoya, y que, por lo tanto, obliguen a realizar un reparto de beneficios aun cuando el acceso a recursos genéticos hubiera sido anterior a la fecha de entrada en vigor del Protocolo de Nagoya, siempre que los beneficios surgidos con posterioridad al Protocolo se deriven de tales recursos genéticos. Este comportamiento voluntario podría ser útil de cara a mantener con las autoridades del país proveedor una buena relación, basada en la buena fe de ambas partes que facilite la obtención de futuras muestras. ${ }^{54}$

52 Singh Nijar, Gurdial, op. cit., p. 19 y ss.

53 Buck, Matthias y Hamilton, Clare, op. cit., p. 50 y ss.

54 Laird, Sarah A. y Ten Kate, Kerry, "Biodiversity Prospecting: the Commercial Use of Genetic Resources and Best Practice in Benefit-Sharing” en Laird, Sarah A. (ed.), Biodiversity and Traditional Knowledge. Equitable Partnerships in Practice, Londres y Sterling (USA), Earthscan, 2002, p. 269. 
Esta revista forma parte del acervo de la Biblioteca Jurídica Virtual del Instituto de Investigaciones Jurídicas de la UNAM www.juridicas.unam.mx

\section{Problemas en la elección y configuración}

de las medidas de cumplimiento relativas a recursos genéticos

El aspecto más importante en el cual el Protocolo de Nagoya innova con respecto al $\mathrm{CDB}$ es en prever una serie de medidas de cumplimiento que todas las partes deben adoptar a fin de verificar que los recursos genéticos que se utilizan en su propia jurisdicción hayan sido accedidos de conformidad con el consentimiento fundamentado previo de la parte proveedora y se hayan establecido con ella condiciones mutuamente acordadas de acuerdo a lo especificado en la legislación del país proveedor. ${ }^{55}$

Un primer reto que presenta la implementación a nivel nacional de medidas de cumplimiento es la selección y adopción de medidas que sean efectivas. Si bien durante las negociaciones se propusieron principalmente cuatro: el requerimiento de divulgación del origen de los recursos genéticos durante el procedimiento de solicitud de patentes, los certificados de origen, medidas de frontera y la introducción de un requisito que obligue a demostrar la legalidad de los recursos al demandar financiamiento público para actividades de investigación, el Protocolo no se decantó por ninguna. Consiguientemente, las partes tienen libertad para decidir.

Sin embargo, ello no debe llevar a pensar que no existen condicionamientos en la elección de las medidas, pues el Protocolo exige que éstas sean "apropiadas, eficaces y proporcionales," ${ }^{56}$ es decir, adecuadas y capaces de lograr un efectivo control sobre la legalidad de los recursos genéticos que son utilizados dentro de la propia jurisdicción. Por lo tanto, es evidente que no sería suficiente el establecimiento de una mera obligación que prescribiera respetar los marcos nacionales de acceso de otro país o de una simple prohibición que mandara no transgredir la ley extranjera; sino que las medidas a adoptar deberían ser capaces de asegurar el correcto estatus legal de los recursos genéticos utilizados en la propia jurisdicción y desalentar efectivamente el incumplimiento. ${ }^{57}$ Asimismo, parece recomendable que al

\footnotetext{
55 Artículo 15.1 Protocolo de Nagoya.

56 Idem.

57 En relación con esto, el Protocolo establece que las Partes aborden de forma apropiada, eficaz y proporcional el incumplimiento o infracción a las medidas de cumplimiento adoptadas. Artículo 16.2 Protocolo de Nagoya. Con ello se piensa que el Protocolo ha querido que las Partes ejerzan su potestad sancionadora por lo cual deberían prever casos/situaciones de incumplimiento (tipificar situaciones de infracción a la ley extranjera en el propio ordena-
} 
no existir definición de lo que debe entenderse por "apropiadas, eficaces y proporcionales", las partes expresen a través de sus Decisiones, criterios objetivos y claros para medir la eficacia de las medidas establecidas por los países al respecto.

Un segundo reto en el marco de la implementación nacional de medidas de control surge en relación con la obligación de designar un punto de verificación a fin de recolectar o recibir información pertinente relacionada con el consentimiento fundamentado previo, la fuente del recurso genético, el establecimiento de condiciones mutuamente acordadas y/o con la utilización de recursos genéticos. ${ }^{58}$ Mediante dicha obligación, el Protocolo de Nagoya introduce el famoso requisito de divulgación, más conocido por el término en inglés disclosure requirement. ${ }^{59}$ El reto surge en vista de que el Protocolo no especifica cuál debe ser el punto de verificación o control que debe seleccionarse. Éste, por su parte, fue uno de los temas más urticantes de las negociaciones ya que los países en desarrollo exigían un punto de control predeterminado - en la mayoría de los casos se mencionaba la oficina de patentes - ; mientras que los países desarrollados tildaban el sistema de inflexible, poco efectivo e ineficiente.

Si bien el Protocolo no incluyó un listado de posibles puntos de verificación — hasta último momento el texto de la negociación sí lo incluía — sí establece que estos deberán ser eficaces y que deberían tener las funciones pertinentes a la vigilancia y la utilización de recursos genéticos, o la recopilación de información pertinente, entre otras cosas, en cualquier etapa de investigación, desarrollo, innovación, pre-comercialización o comercialización. ${ }^{60}$ La buena fe debería por otro lado, guiar a las partes en la elección y designación del punto de control, ya que el éxito del Protocolo depende en realidad de la efectividad de los puntos de verificación que se designen a nivel nacional.

Un tercer desafío nace en relación con el contenido del permiso, una pieza clave entre las medidas de cumplimiento. Éste sirve como prueba de

miento jurídico) y las consecuencias jurídicas (sanciones, etcétera) que acarreará el incumplimiento de las normas de acceso y reparto de beneficios de otro país previsto ahora como infracción a la ley propia.

58 Artículo 17.1.a.i Protocolo de Nagoya.

59 Bajo debate en la Organización Mundial de la Propiedad Intelectual y en la Organización Mundial de Comercio.

60 Artículo 17.1.a.iv Protocolo de Nagoya. 
la decisión de otorgar el consentimiento fundamentado previo y de que se han establecido condiciones mutuamente acordadas ${ }^{61}$ y se convierte más tarde en el "certificado de cumplimiento reconocido internacionalmente" una vez que es notificado al Centro de Intercambio de Información sobre Acceso y Participación en los Beneficios. ${ }^{62}$ El desafío surge porque si bien el Protocolo establece el contenido mínimo del permiso: a) autoridad emisora; $b$ ) fecha de emisión; c) el proveedor; d) identificador exclusivo del certificado; e) la persona o entidad a la que se otorgó el consentimiento fundamentado previo; $f$ ) asunto o recursos genéticos cubiertos por el certificado; g) confirmación de que se han establecido condiciones mutuamente acordadas; $h$ ) confirmación de que se obtuvo el consentimiento fundamentado previo, e i) utilización comercial o de índole no comercial, ${ }^{63}$ resulta evidente que es necesario trabajar aún más en la definición de su contenido a fin de que exista una total armonización al momento de emitir tal certificado. Esta tarea, sin embargo, deberá ser llevada a cabo por la Conferencia de las partes a través de sus Decisiones.

Finalmente, un último desafío para la implementación nacional del Protocolo de Nagoya se origina en la necesidad de vincular en la práctica las obligaciones de los artículos 15.2, que manda a abordar las situaciones de incumplimiento que se produzcan con respecto a las medidas de cumplimiento dictadas, y 17.1(a) que obliga a designar un punto de verificación. Con respecto a ello, caben dos posibles enfoques. Por un lado, como el Protocolo exige establecer al menos un punto de verificación en el que se ha de aportar información acerca de la fuente y el título del recurso genético, lo que se hará mediante la presentación del permiso, y como las partes también están obligadas a abordar situaciones de incumplimiento a la presentación del permiso, una de las más lógicas consecuencias ante tal incumplimiento es la paralización de la tramitación de la solicitud que se estuviere gestionando en el punto de verificación que finalmente se hubiera designado. Acto seguido, dicha falta de acreditación del origen del recurso, se pondría en conocimiento de las autoridades nacionales pertinentes, la parte que otorga el consentimiento fundamentado previo y del Centro de

\footnotetext{
61 Artículo 17.3 y 6.3.e del Protocolo de Nagoya.

62 Artículo 17.2 del Protocolo de Nagoya.

63 Artículo 17.4 del Protocolo de Nagoya.
} 
Intercambio de Información. ${ }^{64}$ Ante esto, cualquiera de ellas podría "accionar el gatillo" e iniciar un procedimiento en el país usuario contra la persona física o jurídica que hubiera incumplido el marco nacional de ABS del país proveedor. La otra posibilidad es la integración de ambos procedimientos en el punto de verificación, de manera que no sólo no se permitiera el procesamiento de solicitudes cuando se comprobara el incumplimiento de los marcos nacionales de ABS, sino que también se establecieran e iniciaran los mecanismos adecuados frente a posibles fraudes o situaciones de incumplimiento. Nada en el Protocolo impide hacerlo de esta segunda manera.

\section{CONCLUSIONES}

El Protocolo de Nagoya surge como una valiosa oportunidad para impulsar la implementación de regímenes efectivos sobre ABS a nivel nacional. Mediante ellos se podría promover la conservación de los recursos genéticos, achicar la brecha biotecnológica existente entre los países en desarrollo y desarrollados, y compensar a las comunidades indígenas y locales por el acceso a su conocimiento tradicional.

El texto del instrumento internacional, sin embargo, presenta una serie de desafíos a la hora de ser implementado a nivel nacional. Este artículo ha individualizado retos relacionados con el ámbito material, dado que los elementos regulados por el Protocolo de Nagoya — recursos genéticos, beneficios derivados de su utilización, derivados, etcétera- son nociones complejas, dinámicas y polémicas. También existen retos en relación con la retroactividad de sus disposiciones, y en relación con ello, al tratamiento que debe darse a los beneficios provenientes de la utilización de los recursos genéticos que se producen con posterioridad a la entrada en vigor del Protocolo pero que se han originado a partir de un acceso anterior a él. Por último, también supone un gran desafío adoptar medidas de cumplimiento y designar un punto de verificación que resulten efectivos y eficientes. En consideración a estos retos, el artículo ha aportado algunos elementos de análisis, notas aclaratorias y posibles soluciones para

64 Artículo 17.1.a.iii del Protocolo de Nagoya. 
Esta revista forma parte del acervo de la Biblioteca Jurídica Virtual del Instituto de Investigaciones Jurídicas de la UNAM

cada uno de ellos, de modo de allanar la adopción e implementación del Protocolo de Nagoya a nivel nacional.

\section{BIBLIOGRAFÍA}

AguiLAR, Gretel, "Access to Genetic Resources and Protection of Traditional Knowledge in the Territories of Indigenous Peoples", Environmental Science \& Policy, 2001, vol. 4, núm. 4-5.

BoIsvert, Valerie y VIVIEN, Franck-Dominique, "The Convention on Biological Diversity: A Conventionalist Approach”, Ecological Economics, 2005, vol. 53, núm. 4.

Buck, Matthias y Hamilton, Clare, "The Nagoya Protocol on Access to Genetic Resources and the Fair and Equitable Sharing of Benefits Arising from their Utilization to the Convention on Biological Diversity", Review of European Community and International Environmental Law, 2011, vol. 20, núm. 1.

Chishakwe, Nyasha y Young, Tomme Rosanne, Access to Genetic Sesources, and Sharing the Benefits of their Use: International and Subregional Issues [Informe inédito], IUCN, 2003, disponible en: http://weavingaweb.org/absdocuments/eng_SADC.pdf.

Convención de Viena sobre el Derecho de los Tratados, 23 de mayo de 1969. UNTS, vol. 1155, 1-18232, disponible en: http://www.wipo.int/ex port/sites/www/wipolex/es/glossary/vienna-convention-es.pdf.

Convenio sobre la Diversidad Biológica, 5 de junio de 1992. UNTS, vol. 1760, I-30619, disponible en: https://treaties.un.org/doc/Treaties/1992/06/19920605\%2008-44\%20PM/Ch_XXVII_08p.pdf.

Decisión II/ 11 de la Conferencia de las Partes del Convenio sobre la Diversidad Biológica, disponible en: http://www.cbd.int/decision/cop/default. shtml?id=7084.

Dickson, David y Jayaraman, K. S., "Aid Groups Back Challenge Patent to Neem Patents”, Nature, 1995, vol. 377.

Directrices de Bonn sobre Acceso a los Recursos Genéticos y Participación Justa y Equitativa en los Beneficios Provenientes de su Utilización. 7 al 19 de abril de 2002. UNEP/CBD/COP/VI/24, disponible en: http: / / www.cbd.int/doc/publications/cbd-bonn-gdls-es.pdf. 
Esta revista forma parte del acervo de la Biblioteca Jurídica Virtual del Instituto de Investigaciones Jurídicas de la UNAM

Documento de la Conferencia de las Partes del Convenio sobre la Diversidad Biológica UNEP/CDB/COP/6/INF/33: Declaración de Cancún de Países Mega-Diversos, disponible en: http://www.cbd.int/doc/meetings/cop/cop-06/information/cop-06-inf-33-es.pdf.

Documento del Grupo de Trabajo Especial de Composición Abierta sobre Acceso y Participación en los Beneficios UNEP/CBD/WG-ABS/7/2: Report of the Meeting of the Group of Legal and Technical Experts on Concepts, Terms, Working Definitions and Sectoral Approaches, disponible en: https://www.cbd.int/doc/meetings/abs/abswg-07/official/abswg07-02-en.pdf.

Documento del Grupo de Trabajo Especial de Composición Abierta sobre Acceso y Participación en los Beneficios UNEP/CBD/WG-ABS/9/ INF/1: The Concept of "Genetic Resources" in the Convention on Biological Diversity and how it Relates to a Functional International Regime on Access and Benefit-Sharing, disponible en: https://www.cbd.int/doc/ meetings / abs / abswg-09/information/abswg-09-inf-01-en.pdf.

GlowkA, Lyle et al., A Guide to the Convention on Biological Diversity, Gland y Cambridge, IUCN, 1994.

GodT, Christine, "Enforcement of Benefit-Sharing Duties in User Countries", en Kamau, Evanson C. y WinTer, Gerd (eds.), Genetic Resources, Traditional Knowledge \& the Law. Solutions for Access and Benefit Sharing, Londres y Sterling (USA), Earthscan, 2009.

Greiber, Thomas et al., Guía explicativa del Protocolo de Nagoya sobre Acceso y Participación en los Beneficios, Gland (Suiza), UICN2012.

Jonge, Bram de y LOUWAars, Niels, "The Diversity of Principles Underlying the Concept of Benefit-Sharing”, en KAMAU, Evanson C. y WINTER, Gerd (eds.), Genetic Resources, Traditional Knowledge \& the Law. Solutions for Access and Benefit Sharing, Londres y Sterling (USA), Earthscan, 2009.

KLOPPENBURG, Jack Jr., "Biopiracy, Witchery, and the Fables of Ecoliberalism”, Peace Review, 2000, vol. 12, núm. 4.

LAIRD, Sarah A. y Ten Kate, Kerry, "Biodiversity Prospecting: the Commercial Use of Genetic Resources and Best Practice in Benefit-Sharing", en Laird, Sarah A. (ed.), Biodiversity and Traditional Knowledge. Equitable Partnerships in Practice, Londres y Sterling (USA), Earthscan, 2002.

Lewis-Lettington, Robert et al., Methodology for Developing Policies and Laws for Access to Genetic Resources and Benefit Sharing, Roma, International Plant Genetic Resources Institute, 2006, disponible en: http: / /www.bio- 
Esta revista forma parte del acervo de la Biblioteca Jurídica Virtual del Instituto de Investigaciones Jurídicas de la UNAM

versityinternational.org/uploads/tx_news/Methodology_for_developing_poli cies_and_laws_for_access_to_genetic_resources_and_benefit_sharing_1150. $p d f$.

Mooney, Pat Roy, "Why I Call it Biopiracy", en Svarstad, Hanne y DHILLION, Shivcharn (eds.), Responding to Bioprospecting: from Biodiversity in the South to Medicines in the North, Oslo, Spartacus Press, AS, 2000.

Plan de Implementación de la Cumbre Mundial de Desarrollo Sostenible de 2002 de Johannesburgo, disponible en: http: / / www.unctad.org/sp/docs / / aconf199d20\&c1_sp.pdf.

Protocolo de Nagoya sobre acceso a los recursos genéticos y participación justa y equitativa en los beneficios que se deriven de su utilización al Convenio sobre la Diversidad Biológica, 29 de octubre de 2010, disponible en: https: / /treaties.un.org/doc/Treaties/2010/11/20101127\%20 02-08\%20PM / XXVII-8-b-Corr-Original.pdf.

SILVESTRI, Luciana, La obligación de participación justa y equitativa en los beneficios derivados de la utilización de recursos genéticos establecida en el protocolo de Nagoya: ¿una oportunidad de desarrollo para países ricos en biodiversidad?, [Tesis doctoral], Madrid, Universidad Rey Juan Carlos, 2014.

SingH NijAR, Gurdial, "The Nagoya Protocol on Access and Benefit Sharing of Genetic Resources: Analysis and Implementation Options for Developing Countries", South Center Research Papers, 2011, núm. 36, http:// www.southcentre.int/wp-content/uploads/2013/05/RP36_The-Nogoya-Protocol_EN.pdf.

SChuster, Brian, "A New Integrated Program for Natural Product Development and the Value of an Ethnomedical Approach", The Journal of Alternative and Complementary Medicine, vol. 7, núm. S1, 2001.

Tsioumani, Elsa, "Access and Benefit Sharing: the Nagoya Protocol", Environmental Policy and Law, vol. 40, núm. 6, 2010.

WinTer, Gerd, "Towards Common Pools of GRs-Improving the Effectiveness and Justice of ABS”, en Kamau, Evanson C. y WinTER, Gerd (eds.), Genetic Resources, Traditional Knowledge \& the Law. Solutions for Access and Benefit Sharing, Londres y Sterling (USA), Earthscan, 2009. 